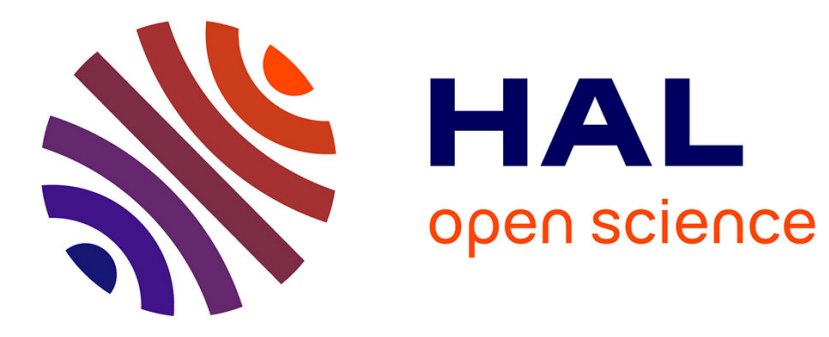

\title{
Individual voxel-based analysis of gray matter in focal cortical dysplasia.
}

Olivier Colliot, Neda Bernasconi, Najmeh Khalili, Samson B Antel, Véronique Naessens, Andrea Bernasconi

\section{To cite this version:}

Olivier Colliot, Neda Bernasconi, Najmeh Khalili, Samson B Antel, Véronique Naessens, et al.. Individual voxel-based analysis of gray matter in focal cortical dysplasia.. NeuroImage, 2006, 29 (1), pp.162-71. 10.1016/j.neuroimage.2005.07.021 . hal-01259130

\section{HAL Id: hal-01259130 \\ https://hal.inria.fr/hal-01259130}

Submitted on 27 Oct 2016

HAL is a multi-disciplinary open access archive for the deposit and dissemination of scientific research documents, whether they are published or not. The documents may come from teaching and research institutions in France or abroad, or from public or private research centers.
L'archive ouverte pluridisciplinaire HAL, est destinée au dépôt et à la diffusion de documents scientifiques de niveau recherche, publiés ou non, émanant des établissements d'enseignement et de recherche français ou étrangers, des laboratoires publics ou privés. 


\section{Individual voxel-based analysis of gray matter in focal cortical dysplasia}

O. Colliot, PhD; N. Bernasconi, MD, PhD; N. Khalili, MSc; S.B. Antel, PhD; V.

Naessens; A. Bernasconi, MD

Department of Neurology and Neurosurgery and McConnell Brain Imaging Center, Montreal Neurological Institute, McGill University, Montreal, Quebec, Canada

Key Words: epilepsy; focal cortical dysplasia; voxel-based morphometry; magnetic resonance imaging; image analysis

Acknowledgments: This work was supported by a grant of the Canadian Institutes of Health research (CIHR-grant \#203707- MOP 57840) and by the Scottish Rite Charitable Foundation of Canada. O.C. is recipient of the Epilepsy Canada Clinical Sciences fellowship. The authors are grateful to Dr F. Andermann and F. Dubeau for referring patients to the study.

\section{Correspondence to:}

Andrea Bernasconi, MD

Montreal Neurological Hospital and Institute

3801 University Street

Montreal, Quebec, Canada H3A 2B4

Tel.: (514)-398-3361

Fax: (514)-398-2975

E-Mail: andrea@bic.mni.mcgill.ca 


\begin{abstract}
High-resolution MRI of the brain has made it possible to identify focal cortical dysplasia (FCD) in an increasing number of patients. There is evidence for structural abnormalities extending beyond the visually identified FCD lesion. Voxel-based morphometry (VBM) has the potential of detecting both lesions and extra-lesional abnormalities because it performs a whole brain voxel-wise comparison. However, on T1-weighted MRI, FCD lesions are characterized by a wide spectrum of signal hyperintensity that may compromise the results of the segmentation step in VBM. Our purpose was to investigate grey matter (GM) changes in individual FCD patients using voxel-based morphometry (VBM). In addition, we sought to assess the performance of this technique for FCD detection with respect to lesion intensity using an operator designed to emphasize areas of hyperintense T1 signal. We studied 27 patients with known FCD and focal epilepsy, and 39 healthy controls. We compared the GM map of each subject (controls and patients) with the average GM map of all controls and obtained a GM z-score map for each individual. The protocol being designed to achieve a maximal specificity, no differences in GM concentration were found in the control group. The z-score maps showed an increase in GM that coincided with the lesion in 21/27 (78\%) patients. Five of the six remaining patients whose lesions were not detected by VBM, presented with a strong lesion hyperintensity and a significant part of their lesion was misclassified as white matter. In 16/27 (59\%) patients, there were additional areas of GM increase distant from the primary lesion. Areas of GM decrease were found in 8/27 (30\%) patients. In conclusion, individual voxel-based analysis was able to detect FCD in a majority of patients. Moreover, FCD was often associated with widespread GM changes extending beyond the visible lesion. In its current form, however, individual VBM may be unable to detect lesions characterized by strong signal intensity abnormalities.
\end{abstract}




\section{Introduction}

Malformations caused by abnormalities of cortical development (MCD)(Barkovich et al., 2001) have been increasingly recognized as an important cause of intractable focal epilepsy. Taylor-type focal cortical dysplasia (FCD) (Taylor et al., 1971), a pathology due to abnormal neuroglial proliferation, is the most frequent MCD in patients referred for presurgical evaluation (Frater et al., 2000, Sisodiya, 2000). The pathologic spectrum of FCD includes various degrees of cortical thickening, indistinctness ("blurring") of the gray matter (GM)-white matter (WM) transition, abnormal sulcal pattern, abnormal neuroglial elements in the underlying WM, and disruption of the normal cortical lamination associated with an excess of large, aberrant neurons (Prayson et al., 2002).

On T1-weighted MRI, FCD is mainly characterized by variable degrees of GM thickening, a poorly defined transition between GM and WM, and hyperintense signal within the dysplastic lesion relative to normal cortex (Barkovich and Kuzniecky, 1996, Lee et al., 1998). High-resolution MRI of the brain has made it possible to identify FCD in an increasing number of patients (Barkovich and Kuzniecky, 1996, Grant et al., 1998) . However, in many patients, lesions of FCD are characterized by minor structural abnormalities that go unrecognized or are too subtle to be detected by standard radiological analysis.

In FCD, there is evidence for structural abnormalities extending beyond the visually identified lesion. Histological abnormalities may be disseminated rather than confined to a single patch or even be multifocal (Frater et al., 2000, Gomez-Anson et al., 2000, Prayson et al., 2002, Taylor et al., 1971). Electrophysiological studies in animal models of MCD (Jacobs et al., 1999, Kraemer et al., 2001, Redecker et al., 1998) and humans have also shown that the epileptogenic region frequently exceeds the boundaries of the obvious lesion (Palmini et al., 1995, Raymond and Fish, 1996, Tassi et al., 2001). The presence of these widespread structural abnormalities may be an important factor explaining the relatively poor outcome of surgery in MCD patients (Sisodiya, 2000) when compared to patients with other causes of epilepsy such as hippocampal sclerosis (Engel, 1996).

To date only few studies have been dedicated to the automatic detection of FCD and to the evaluation of structural changes distant to the primary lesion using quantitative methods that can potentially capture structural changes too subtle to be detected by visual inspection (Sisodiya et al., 1995, Sisodiya and Free, 1997). We previously showed that computational models based on known MRI features of FCD and texture analysis improve the sensitivity of detection of FCD relative to the standard MRI evaluation (Antel et al., 2002, Antel et al., 2003, Bernasconi et al., 2001).

Voxel-based morphometry (VBM) is a fully automated technique allowing identification of regional differences in the amount of GM and WM in spatially standardized structural MR images, enabling an objective analysis between groups of subjects (Ashburner and Friston, 2000). While originally developed for group comparison, VBM has also been used to assess morphological changes in individual brains compared to a control group (Mehta et al., 2003, Mummery et al., 2000, Wilke et al., 2003, Woermann et al., 1999). As VBM involves a whole brain voxel-wise comparison, it has the potential of detecting both the FCD lesion and structural abnormalities that would extend beyond the lesion itself. However, accuracy and sensitivity of VBM analysis depend upon the quality of the underlying preprocessing steps, namely segmentation and spatial normalization. When dealing with brain 
pathologies, a major source of error could arise when abnormal voxel intensities are present (Mehta et al., 2003). In our previous studies, we developed a computational operator to model the hyperintense signal within the FCD lesion (Antel et al., 2002, Bernasconi et al., 2001) and proved that FCD lesions are characterized by a wide spectrum of hyperintense voxels with respect to normal cortex (Colliot et al., 2004). Thus, it is possible that FCD may be misclassified as WM instead of GM, and therefore be undetected by VBM.

Our purpose was to investigate GM changes in individual FCD patients using VBM. In addition, we sought to assess the performance of this technique for FCD detection with respect to lesion intensity. 


\section{Methods}

\section{Subjects}

We studied 27 adult patients (11 males, mean age \pm [standard deviation (SD)] $=25 \pm 7$ years) who had been investigated at the Montreal Neurological Institute for partial epilepsy related to FCD and had high-resolution MRI, including curvilinear reformatting (Bastos et al., 1999). The FCD lesion was seen on MRI in all of them. Patients were compared to 39 sex- and age-matched healthy controls (18 males, mean age $29 \pm 8$ ). The Ethics Board of the Montreal Neurological Institute and Hospital approved the study and written informed consent was obtained from all participants.

Eighteen patients $(18 / 27=67 \%)$ underwent surgery and FCD was confirmed by histopathology in all of them. Mean post-operative follow-up was 4.7 years (median = 4.5 years). Ten patients $(10 / 18=56 \%)$ became completely seizure free after surgery (Engel's classification IA) (Engel et al., 1993), while eight others did not achieve complete seizure freedom (five had rare disabling seizures -Engel's classification II-, two had a worthwhile improvement -Engel's classification III- and one had no worthwhile improvement -Engel's classification IV-). Three patients refused the surgical intervention and another patient with normal neurological examination was not operated because the FCD lesion encroached the sensorimotor hand area. In five patients, seizures were controlled with antiepileptic medication.

The FCD lesions were located in the frontal lobe in 17/27 (63\%) patients and in the parietal lobe in 10/27 (37\%) patients.

\section{MRI scanning}

In all patients and healthy controls MRI volumetric images were acquired on a $1.5 \mathrm{~T}$ Gyroscan (Philips Medical System, Best, The Netherlands) using a T1-fast field echo sequence $\left(\mathrm{TR}=18, \mathrm{TE}=10,1\right.$ acquisition average pulse sequence, flip angle $=30^{\circ}$, matrix size $=256 \times 256$, FOV $=256$, thickness $=1 \mathrm{~mm}$ ).

\section{Image processing}

Voxel-wise processing of the MRI included the following steps (Figure 1). Images were automatically registered into a standard, stereotaxic space to adjust for differences in total brain volume and brain orientation (Collins et al., 1994). Each image underwent automated correction for intensity non-uniformity and intensity standardization (Sled et al., 1998). This correction produces consistent relative gray matter (GM), white matter (WM) and cerebro-spinal fluid (CSF) intensities. Classification of brain tissue in GM, WM and CSF was performed using the INSECT algorithm (Zijdenbos et al., 1998), which relies on an artificial neural network classifier. GM masks were then blurred with an isotropic Gaussian kernel of $12 \mathrm{~mm}$ FWHM to generate 3D-maps of GM "concentration". The validity of a $12 \mathrm{~mm}$ smoothing kernel in unbalanced designs has been shown previously (Salmond et al., 2002). All GM concentration maps from the control group were then averaged to produce mean and standard deviation (SD) maps.

We compared the GM concentration map of each subject (controls and patients) with the average GM map of all controls (Figure 1A) and obtained a GM z-score map for each individual (Figure 1B).

\section{Assessment of FCD hyperintensity}


To assess whether the hyperintensity of FCD lesions (i.e. the fact that they are characterized by higher signal intensity than the normal cortex) has a significant impact on the automatic tissue segmentation and on the results of individual voxel-based analysis, we used our previously developed model of relative intensity (Bernasconi et al., 2001). Relative intensity is defined for a particular voxel as $100 *[\mathrm{Bg}-|\mathrm{Bg}-\mathrm{g}(\mathrm{i}, \mathrm{j}, \mathrm{k})|] / \mathrm{Bg}$, where $\mathrm{Bg}$ is the intensity at the boundary between $\mathrm{GM}$ and $\mathrm{WM}$, and $\mathrm{g}(\mathrm{i}, \mathrm{j}, \mathrm{k})$ represents the intensity at a given voxel (Figure 2A). Using the relative difference enables the analysis of voxels located within cortical GM in which the intensity is higher than normal and may even overlap that of the WM.

The primary FCD lesions were segmented manually on the high-resolution T1weighted MRI by a trained rater (V.N.). The proportion of FCD lesion label classified as GM and WM was calculated. We then computed the mean relative intensity within the segmented FCD (Figure 2).

\section{Analysis}

To detect significant differences in GM concentration, a voxel was considered abnormal if its value fell outside the mean of the controls $\pm 4.5 \mathrm{SD}$. This threshold was chosen to exceed the maximum GM increase or decrease seen in any control subject.

The voxel-based analysis was considered to successfully detect the FCD lesion when a GM increase greater than 4.5 was present at the lesion location. To quantify the fraction of the lesion correctly identified by the method, we computed a lesion coverage ratio, which is equal to the percentage of voxels in the lesion label having a GM z-score greater than 4.5. Association between the ratio of lesion coverage and the size of the FCD lesion, as determined by manual segmentation, was assessed using Pearson correlation coefficient (r).

To assess the influence of the chosen threshold (4.5 SD) on the detection results, we computed the sensitivity [percentage of positives correctly identified = true-positives / (true-positives + false-negatives)] and specificity [percentage of negatives correctly identified $=$ true-negative $/$ (true-negatives + false-positives) $]$ for different threshold values (i.e., $5 \mathrm{SD}, 4.5 \mathrm{SD}, 4 \mathrm{SD}, 3.5 \mathrm{SD}, 3 \mathrm{SD}, 2.5 \mathrm{SD}, 2 \mathrm{SD}$ ). True positives are patients that had an increase in GM concentration higher than the threshold and coinciding with the lesion. False positives are healthy controls that had a GM increase larger than the threshold.

To assess the influence of the size of the healthy control group on the results, we randomly sub-sampled the control population into two groups of 20 and 25 subjects.

We considered as extra-lesional GM increase any GM increase greater than our 4.5 threshold and not coinciding with the lesion. Conversely, we considered as GM decreases, regions of GM decrease smaller than -4.5 SD that did not coincide with the lesion.

We assessed using $\chi^{2}$ test whether the presence of extra-lesional GM increases as well as extra-lesional GM decreases differed between patients who became seizure free after surgery compared to those who did not. 


\section{Results}

\section{Lesional GM increase}

The z-score maps showed an increase in GM that coincided with the primary FCD lesion in 21/27 (78\%) patients. Examples of individual patients are presented in Figure 3. In $6 / 27(22 \%)$ patients, no GM increase was found at the FCD location.

FCD lesions volumes ranged from $773 \mathrm{~mm}^{3}$ to $88726 \mathrm{~mm}^{3}$ (mean $=10803 \mathrm{~mm}^{3} \pm$ 16037). For the 21 patients in whom the lesion was detected, the mean lesion coverage ratio was $21 \%(\mathrm{SD}=17 \%$, range $=0.1 \%-63 \%)$. There was no correlation between FCD volume and the coverage ratio. Results of FCD lesion detection corresponding to various coverage ratios are presented in Figure 3.

The results for sensitivity and specificity obtained for different threshold values are presented in Figure 4.

Using a randomly sub-sampled group of 20 controls and our chosen threshold of 4.5 SD, 22/27 (81\%) lesions were detected and no GM increase above 4.5 was found in the controls. When using 25 controls and the same threshold, 21/27 (78\%) lesions were detected and no increase was found in the controls.

\section{Extra-lesional GM increase}

Sixteen $(16 / 27=59 \%)$ patients had extra-lesional areas of GM increase. This was the case for $13 / 21(62 \%)$ of those who presented a GM increase at the location of the lesion and for three $(3 / 6=50 \%)$ of those whose lesion was not detected by VBM. For the 16 patients with extra-lesional GM increase, the mean number of areas of increase was 2 (range $=1$ 4). There was no difference in extra-lesional GM increases between patients who became seizure free after surgery and those who did not (50\% vs. 50\%).

Four $(4 / 16=25 \%)$ patients had extra-lesional GM increases in the same hemisphere as the lesion; four $(4 / 16=25 \%)$ had $\mathrm{GM}$ increases in the hemisphere contralateral to the FCD lesion; and eight $(8 / 16=50 \%)$ had bilateral GM increases. The areas of GM increase were predominantly found in the frontal lobe (10 patients). Other locations included: temporal (5), occipital (5), parietal (3) lobes, and the insula (3). Examples of FCD patients with extra-lesional GM increases are presented in Figure 5.

\section{Extra-lesional GM decrease}

Areas of GM decrease were found in eight $(8 / 27=30 \%)$ patients. There was no difference in extra-lesional GM decrease between patients who became seizure free after surgery and those who did not $\left(3 / 10=30 \%\right.$ vs $\left.4 / 8=50 \% \chi^{2}=0.75, \mathrm{df}=1, \mathrm{p}=0.38\right)$.

Five patients $(5 / 8=63 \%)$ had GM decrease in the same hemisphere as the FCD lesion; one patient $(1 / 8=13 \%)$ had GM decrease in the hemisphere contralateral to the lesion; and two $(2 / 8=25 \%)$ had bilateral GM decrease. The majority of patients $(6 / 8=75 \%)$ had GM decrease in the temporal lobe neocortex. Other areas included: frontal (3), occipital (2), and the insula (2). Examples of patients with extra-lesional GM decrease are presented in Figure 6.

\section{Performance of VBM with respect to FCD hyperintensity}

Relative intensity values for FCD patients are presented in Table 1. The relative intensity computed over the whole lesion label ranged between 72 and 93 in our patients with a mean of $85 \pm 4.6$ (arbitrary units). 
In 5/6 patients in whom VBM was unable to identify the primary lesion, the FCD was strongly hyperintense (relative intensity values ranging from 87 to 93 ). In fact, four of them presented with the four highest hyperintensity values in the patient group. These five patients also had a lower portion of the lesion classified as GM (from 37\% to $80 \%$ ). Representative examples are presented in Figure 7. 


\section{Discussion}

Using individual voxel-based analysis, we were able to reliably and automatically detect the FCD lesion in more than three-quarter of our patients.

\section{Comparison with previous quantitative methods}

Our VBM approach provided average lesion coverage of $21 \%$ compared to expert tracing. This was slightly higher than that of our previously implemented automatic classifier based on computational models of FCD and texture analysis, which attained 13\% lesion coverage (Antel et al., 2003). The sensitivity of the individual VBM analysis presented here was 78\%. In a previous work based on individual VBM analysis of FCD lesions, the sensitivity for lesion detection was similar to our results (Wilke et al., 2003). In that study, the authors chose an arbitrary threshold of 2 SD above the mean GM concentration in normal controls as indicative of abnormality. Therefore, they did not fully evaluate the specificity of their methodology. Indeed, at a threshold of 2 SD, we found numerous areas of false positives in our control population. To detect reliably abnormal GM, we selected a higher threshold, so that no areas of GM changes (increase or decrease) could be found in any of our healthy control subjects. Our protocol was thus designed to limit as much as possible the detection of false positives, particularly in patients who undergo presurgical evaluation for medically refractory seizures.

\section{Statistical threshold}

Choosing a threshold value of 4.5 that exceeded the maximum GM change in controls allows taking multiple comparisons into account in an empirical manner. Indeed, the number of voxels, and thus the number of comparisons, are the same when analyzing GM maps in individual patients and controls. Thus, the proportion of voxels that will be found abnormal by chance is also the same in patients and controls. Since no false positives are found in controls at this threshold, the probability of finding a false positive in the patients is very low. Our threshold selection, therefore, constitutes a significant improvement over existing individual VBM analyses that use a 2SD threshold and do not take into account multiple comparisons (Wilke et al., 2003).

A threshold of 4.5 SD was the minimal value to avoid false positives in healthy controls. A slightly lower value of $4 \mathrm{SD}$ would allow the detection of one additional lesion while decreasing the specificity from $100 \%$ to $90 \%$. Thresholds of 4 SD and 4.5 SD lead to high sensitivity and specificity and are thus both equally acceptable when using individual VBM for FCD detection in a clinical context. On the contrary, with lower threshold values, the number of false positives would increase rapidly as shown in Figure 4. In a clinical setting, it is conceivable to explore different thresholds below 4.0 $\mathrm{SD}$, provided that the output of VBM converges with the information given by independent exams such as EEG, clinical semiology and other imaging methods.

\section{Influence of the control group}

The choice of the healthy control sample did not have a significant impact on the results. The only difference was that using 20 controls, one additional lesion could be detected at a threshold of 4.5 SD. However, in spite of this, it is preferable to use a larger group of healthy controls to better cover the spectrum of normal anatomical variability.

\section{Performance of VBM with respect to FCD hyperintensity}


Our VBM method failed to detect the FCD lesion in six patients. With a threshold of 4 $\mathrm{SD}$, one of these six lesions would be detected by VBM. To identify additional lesions, the threshold should be lowered to at least $3 \mathrm{SD}$, leading to a specificity of only $31 \%$.

Even though there was no significant correlation between lesion size and coverage, we failed to detect the two FCD lesions with the smallest volume in the group. It is likely that a very small lesion will correspond to a small focal area of GM increase, which may not exceed the order of magnitude of the normal variability of GM "concentration" in that region. The second plausible factor that can explain the ineffectiveness of VBM is FCD hyperintensity. In five of the undetected FCDs, a relatively small portion of the lesion was classified as GM by the automated segmentation algorithm, even though three of these lesions had a medium to large size compared to the others. In all five cases the FCD lesion was strongly hyperintense relative to the rest of the neocortex. Therefore a large proportion of lesional voxels were misclassified as WM. These findings emphasize the need for caution when using VBM alone to detect pathologies characterized by intensity abnormalities. They also demonstrate that VBM and our computational model of FCD hyperintensity convey complementary information.

\section{Alternative and complementary approaches}

Image analysis approaches other than VBM have the potential to detect lesions by modeling the abnormal characteristics of FCD. We previously developed a classifier that integrates computational models of the main FCD features, such as focal thickening of the cortex computed directly in the 3D image space (Antel et al., 2003). Other approaches relying on surface-based analysis of cortical thickness (Lerch et al., 2004, Sailer et al., 2003) would allow a more direct comparison of cortical areas between subjects. By providing an evaluation of cortical thickness compared to similar regions in healthy controls these methods could improve FCD detection. Furthermore, automatic methods for sulci extraction and analysis have been recently developed (Riviere et al., 2002). These techniques have the potential to identify abnormal sulcal patterns that may also be associated with FCD (Usui et al., 2001a, Yagishita et al., 1997). Overall, these advanced techniques could be used in conjunction with our individual VBM approach. It is however important to keep in mind that GM/WM segmentation constitutes a crucial common pre-processing step in all these approaches. It can thus be expected that signal hyperintensity associated with FCD might still compromise the end results, similarly to what was demonstrated here. To this purpose, future effort should be dedicated to the development of segmentation algorithms that take into account FCD hyperintensity, possibly relying on region-growing techniques (Mangin et al., 1995) and computational models of hyperintensity (Bernasconi et al., 2001).

In our experience, T1-weighted 3-D gradient-echo technique with a voxel size of $1 \times 1 \times 1 \mathrm{~mm}^{3}$ on a 1.5 Tesla scanner provides good anatomic details for digital image processing. High-field magnets are increasingly available in research and clinical facilities. Higher magnetic field-strength provides high signal-to-noise ratio due to higher signal intensity, which allows for increased spatial resolution or faster imaging. However, other unwanted effects such as the sensitivity to magnetic field inhomogeneities and changes in relaxation times increase with higher field strength, which lead to changes in image contrast. High-field imaging has the potential to improve 
lesion detection, provided that appropriate intensity correction methods become available.

\section{Extra-lesional GM changes in FCD}

Focal areas of extra-lesional GM decrease were found in approximately one third of the patients, the majority of them being located within the hemisphere ipsilateral to the primary FCD lesion and in the temporal lobe neocortex. Similar findings were reported in a previous study of 10 patients with various MCD, mostly heterotopias (Woermann et al., 1999). Although the exact nature of GM decrease remains unclear, its location in the temporal lobe ipsilateral to the primary FCD lesion, that was frontal in most patients, could indicate GM atrophy secondary to alteration of fronto-temporal neuronal circuits. This hypothesis is supported by previous observations showing decreased metabolic function in the temporal lobe of patients with extra-temporal lesions, including FCD (Miller et al., 2000).

Fifty-nine percent of our patients exhibited extra-lesional areas of GM increase, which were present in equal proportions in the hemisphere ipsi- and contralateral to the FCD lesion. Areas of GM increase were located mainly within the frontal lobe. Interestingly, the majority of FCD lesions were also located in the frontal lobe. This might indicate that a similar developmental process is the source of both primary FCD lesions and extra-lesional GM increase. Retrospective visual analysis of the areas of GM increase in individual z-score maps and on conventional MRI of these patients was not suggestive of FCD pathology. Moreover, scalp EEG data from these regions did not exhibit any epileptic abnormality nor patterns found to be associated with FCD such as polyspike activity (Gambardella et al., 1996). These findings are in agreement with our previous work on automatic classification of FCD, where we found extra-lesional clusters exhibiting a pattern of features similar to FCD lesions in one third of patients (Antel et al., 2003). The absence of any false positives in our control subjects combined with pathological (Prayson et al., 2002, Taylor et al., 1971) and MRI reports (Sisodiya, 2000, Sisodiya, 2004, Sisodiya et al., 1995, Sisodiya and Free, 1997) of diffuse cortical involvement in FCD suggests that these clusters may indeed indicate abnormal regions that are otherwise undetectable via conventional means.

Areas of GM increase detected by VBM in FCD patients could represent abnormalities of gyration rather than true increase in GM or cortical thickening. On visual analysis of conventional MRI, sulcal and gyral abnormalities are sometimes noted in patients with MCD. These abnormalities are thought to represent an underlying pathological cortical cytoarchitecture (Barth, 1987, Rakic, 1988) and have been described in the proximity of the MRI visible lesion, but also distant from it. Sulcal and gyral abnormalities are characterized by a large spectrum of changes ranging from clefts of various depth (Bronen et al., 2000) to minor changes such as slight shape asymmetry, broad gyri, shallow or deep sulci, altered gyral or sulcal orientation or location (displacement) (Muntaner et al., 1997, Raymond et al., 1995, Usui et al., 2001b, Yagishita et al., 1997). The fact that sulcal and gyral abnormalities have been rarely reported is not surprising since the cerebral cortex is a convoluted 3D structure and abnormalities in its surface gyration may not be appreciated when it is viewed on $2 \mathrm{D}$ MRI slices. Our VBM procedure involved a linear spatial normalization of images, and thus did not attempt to match exactly every gyrus in the brain. Therefore, an abnormality of gyral pattern such as deeper gyri would appear on the VBM maps as an increase in 
GM. Ultimately, correlative pathological studies would be required to shed more light on the nature of extra-lesional GM abnormalities in FCD.

\section{Clinical considerations}

Previous studies have suggested that a less favorable post-surgical outcome in MCD patients might be linked to the presence of extra-lesional pathology (Sisodiya, 2000). Although there have been numerous studies on post-surgical outcome in MCD (CohenGadol et al., 2004, Colombo et al., 2003, Fauser et al., 2004, Francione et al., 2003, Frater et al., 2000, Kral et al., 2003, Prayson and Frater, 2003, Russo et al., 2003, Sisodiya, 2000, Tassi et al., 2002), none of them included a detailed MRI assessment of extralesional brain. Therefore, the above hypothesis has not yet been tested adequately. In our study, we found no influence of extra-lesional GM increase on surgical outcome. However, this findings need to be confirmed in a larger population of surgically treated FCD patients.

In conclusion, using individual voxel-based analysis of GM, we identified the primary FCD lesion in the majority of our patients. We also showed that brain pathology in FCD extends beyond the visible lesion and is associated with widespread GM changes. While VBM has the potential to detect the increase in GM associated with FCD, lesions presenting with signal intensity different from normal GM might be misclassified as WM, and thus not be detected. It can be expected that the combination of VBM with other computational models of FCD could enhance both sensitivity and lesion coverage. 


\section{Tables}

Table 1. Mean and standard deviation of FCD relative intensity, percent classified as GM, lesion volume and maximum increase in GM concentration that coincided with the lesion. Mean values are presented for all FCD patients. Individual values are presented for those six whose lesions were not identified using VBM.

\begin{tabular}{|c|c|c|c|c|c|c|c|}
\hline & \multicolumn{2}{|c|}{$\begin{array}{l}\text { Relative intensity } \\
\text { (arbitrary units) }\end{array}$} & \multicolumn{2}{|c|}{$\begin{array}{l}\% \text { of lesion } \\
\text { classified as GM }\end{array}$} & \multicolumn{2}{|c|}{$\begin{array}{l}\text { Lesion volume } \\
\left(\mathrm{mm}^{3}\right)\end{array}$} & \multirow{2}{*}{$\begin{array}{l}\begin{array}{l}\text { Max GM } \\
\text { increase } \\
(\mathrm{SD})\end{array} \\
8.2 \pm 5.8\end{array}$} \\
\hline $\begin{array}{l}\text { All patients } \\
\text { mean } \pm \text { SD }\end{array}$ & $85 \pm 4.6$ & & $85 \% \pm 1$ & & $10803 \pm$ & 16037 & \\
\hline range & $72-93$ & & $37 \%-9$ & & $773-88$ & 26 & $1.1-22$ \\
\hline $\begin{array}{l}\text { Patients whose } \\
\text { lesion was not } \\
\text { detected by VBM }\end{array}$ & Value & Rank & Value & Rank & Value & Rank & \\
\hline Patient 20 & 93 & 1 & $37 \%$ & 1 & 17267 & 24 & 1.9 \\
\hline Patient 8 & 92 & 2 & $69 \%$ & 3 & 1797 & 4 & 3.0 \\
\hline Patient 17 & 92 & 3 & $48 \%$ & 2 & 5113 & 13 & 1.1 \\
\hline Patient 4 & 91 & 4 & $80 \%$ & 7 & 807 & 2 & 4.1 \\
\hline Patient 24 & 87 & 8 & $77 \%$ & 6 & 5008 & 12 & 2.8 \\
\hline Patient 11 & 84 & 16 & $94 \%$ & 20 & 773 & 1 & 3.1 \\
\hline
\end{tabular}




\section{Figures}
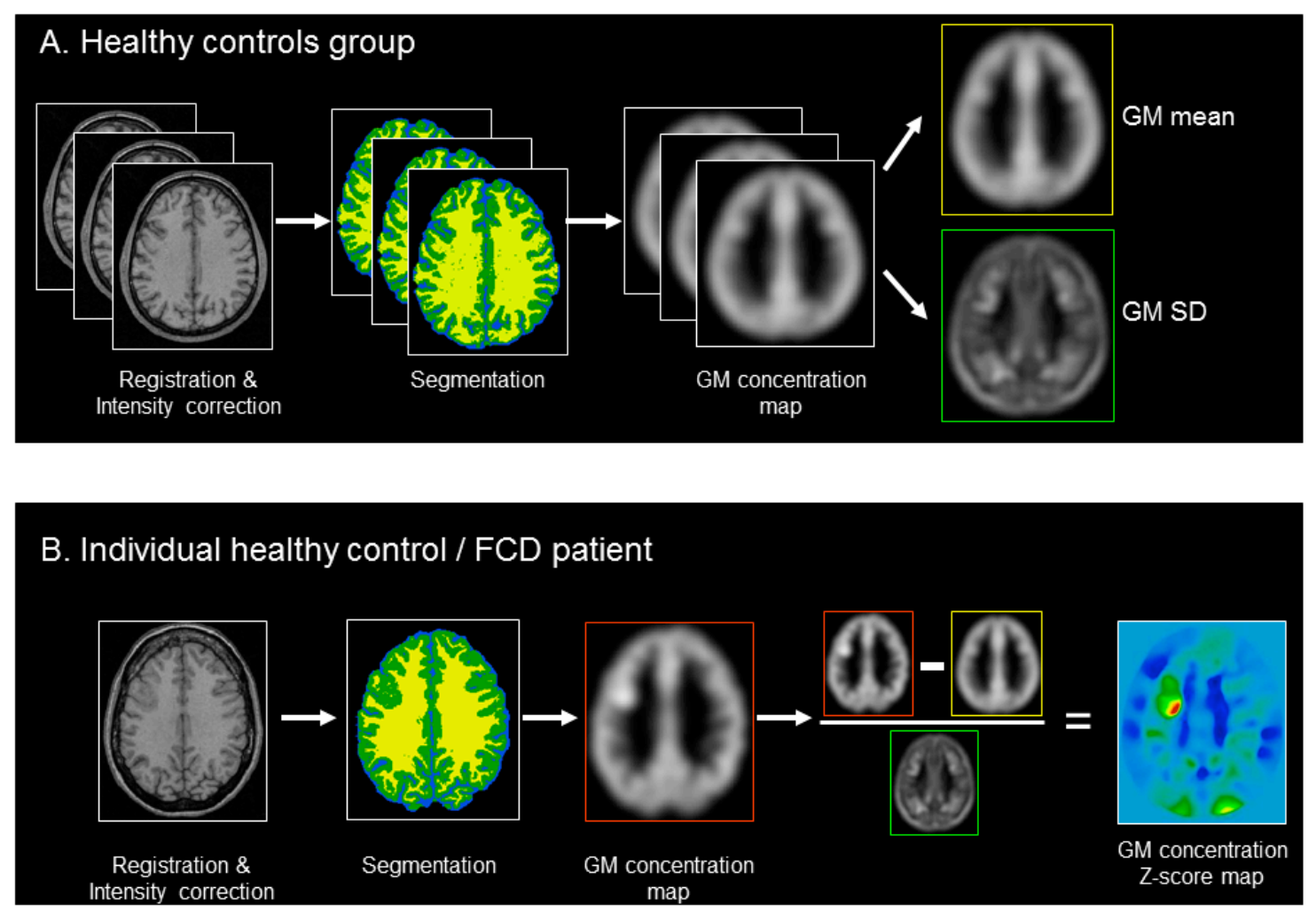

Figure 1. Voxel-wise processing of MR images. (A) Processing steps for the healthy controls group. First, T1-weighted MRI are registered into a standard, stereotaxic space and corrected for intensity non-uniformity. Images are then segmented into GM (green), WM (yellow) and CSF (blue). A GM "concentration" map is then generated (see text for details). All GM concentration maps are then averaged to produce mean and standard deviation maps for the control group. (B) The processing steps used for the analysis of a single individual healthy control or a single FCD patient. A representative patient with FCD is presented. Registration, intensity correction, segmentation and creation of a GM concentration map are done as described in Panel A. Finally, a GM concentration z-score map is computed by subtracting the GM mean of the healthy controls from the GM concentration map of each individual and dividing the result by the GM SD of the healthy controls. 

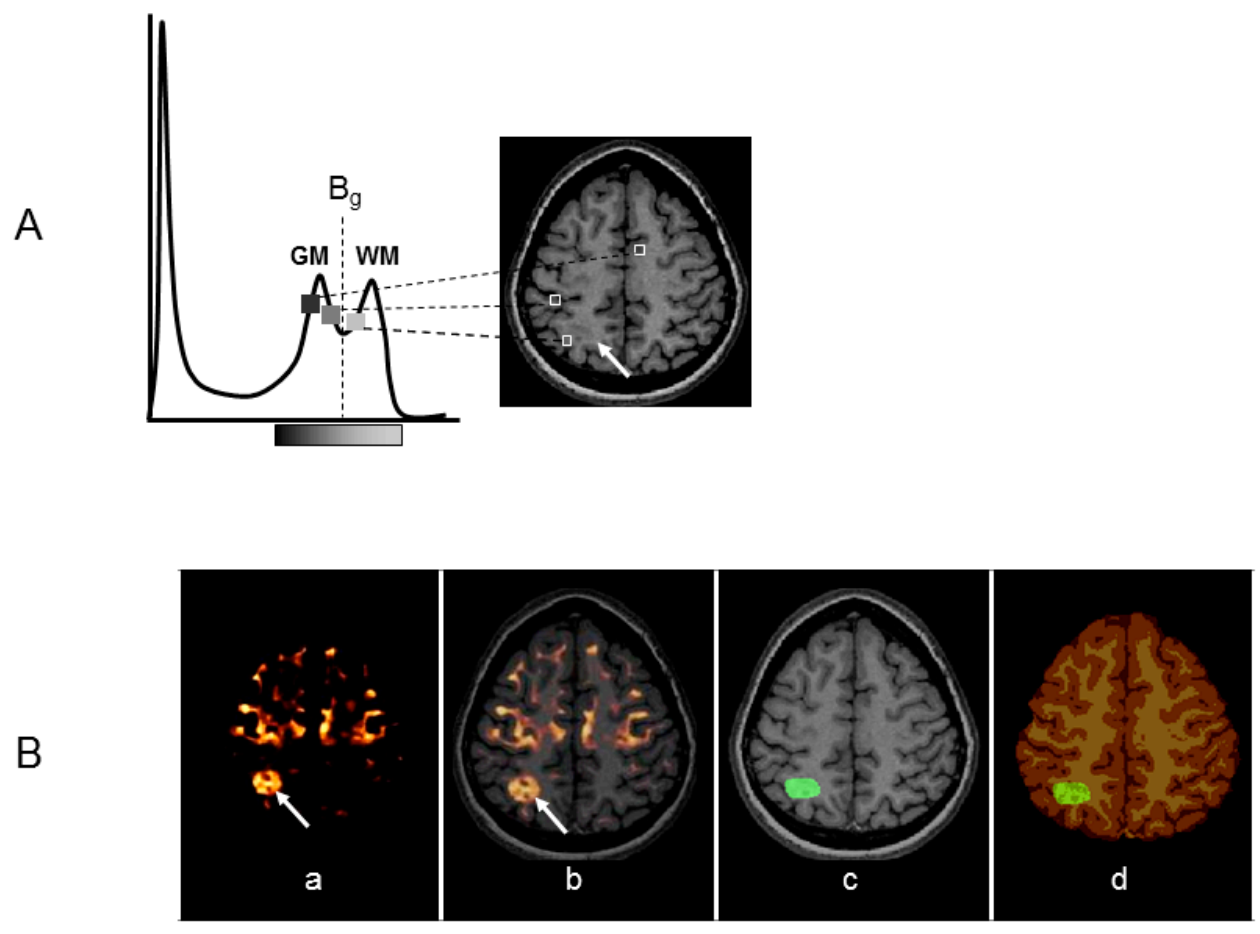

Figure 2. Computation of relative intensity. (A) To model the hyperintense signal within the FCD lesion on T1-weighted MRI, the normalized absolute difference between the intensity at a given voxel and the intensity at the GM/WM boundary $(\mathrm{Bg})$, as defined by the image histogram, is calculated (see text for details). Using the relative difference allows analysis of voxels located within the GM in which the intensity is higher than normal and overlaps that of the WM. GM at higher intensity is closer to the GM-WM boundary. (B) Computation of relative intensity within the manually segmented FCD. The relative intensity map is presented alone (a) and superimposed on the T1-weighed MRI (b). On the relative intensity map, hyperintense voxels appear brighter. The manual lesion label (c) in which relative intensity is calculated is superimposed on the segmented MRI (d). This allows calculating the proportion of FCD lesion label classified as GM and WM. The arrow shows the FCD lesion. 


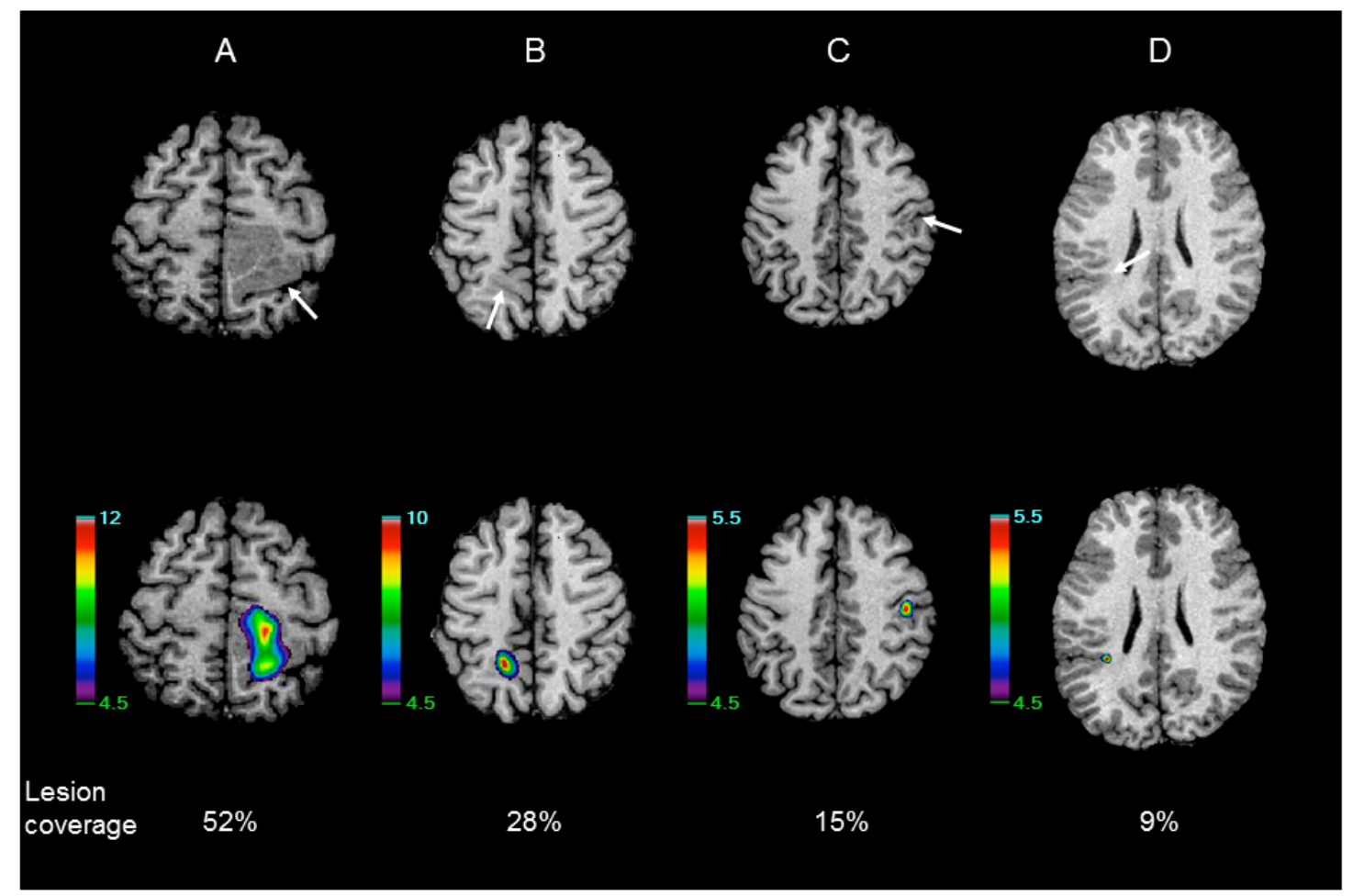

Figure 3. Four examples (A-D) of FCD lesion detection using individual voxel-based analysis of GM. Top panels show the T1-weighted MRI in which the FCD lesion is indicated by an arrow. Bottom panels show the thresholded GM concentration z-score map superimposed on the MRI. The lesion coverage produced by the automated VBM method is indicated for each case (see text for details). 


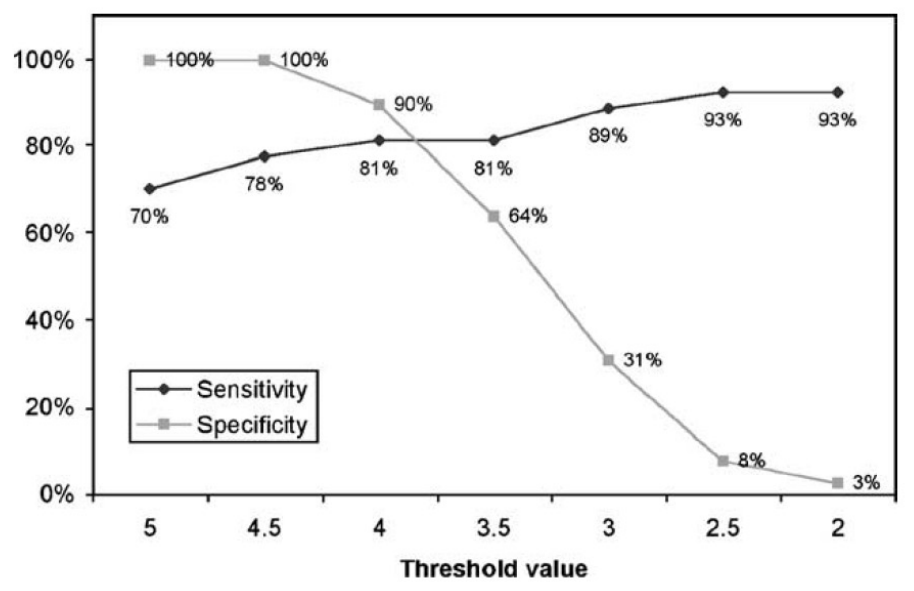

Figure 4. Sensitivity and specificity obtained for different thresholds. 


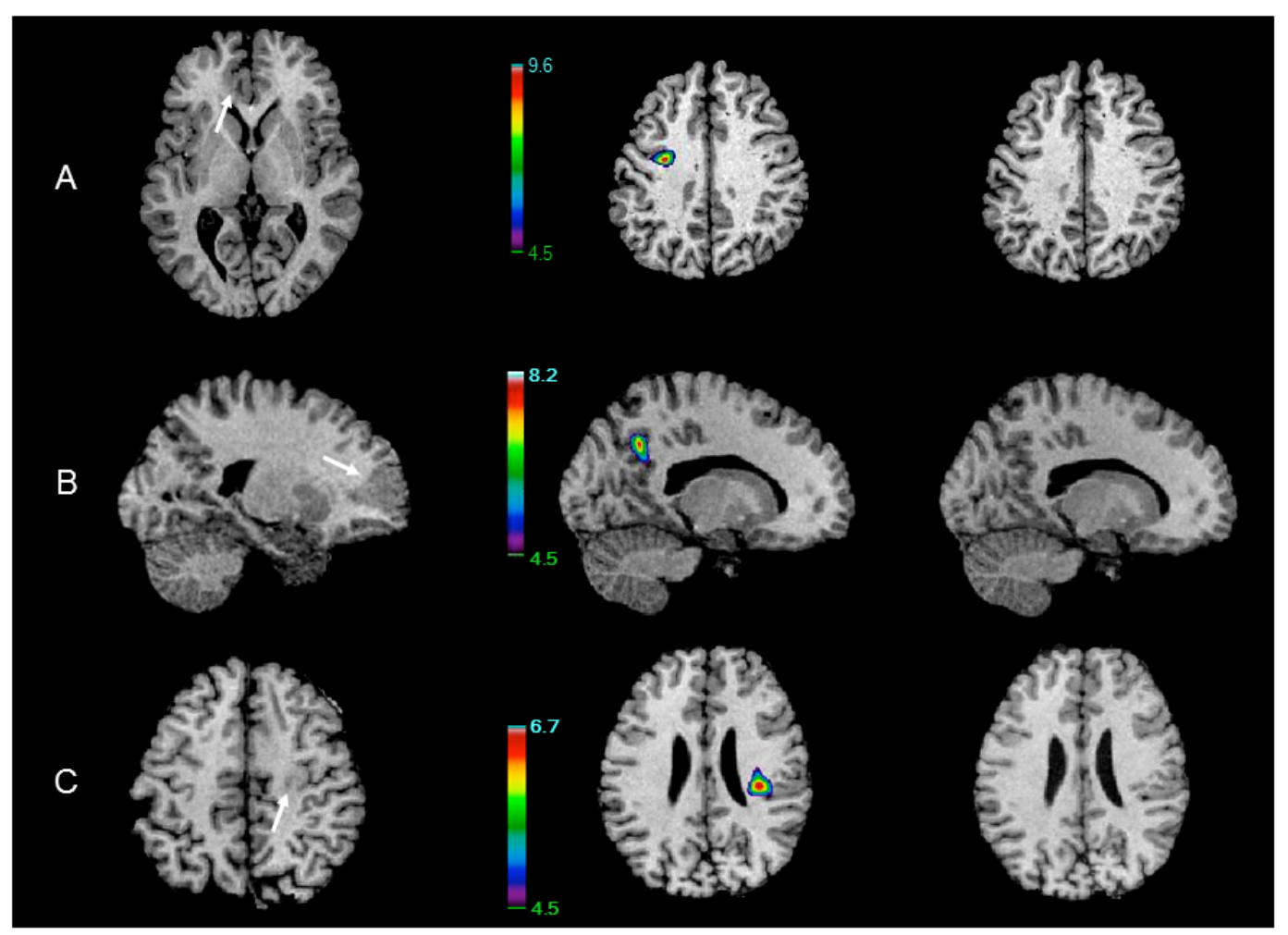

Figure 5. Extra-lesional GM increase in three (A-C) FCD patients. Panel on the left shows the primary FCD lesion on the T1-weighted MRI indicated by an arrow. The middle panel shows the area of extra-lesional GM increase on the GM concentration zscore map superimposed on the MRI, and the right panel shows the T1-weighted MRI at that level. 


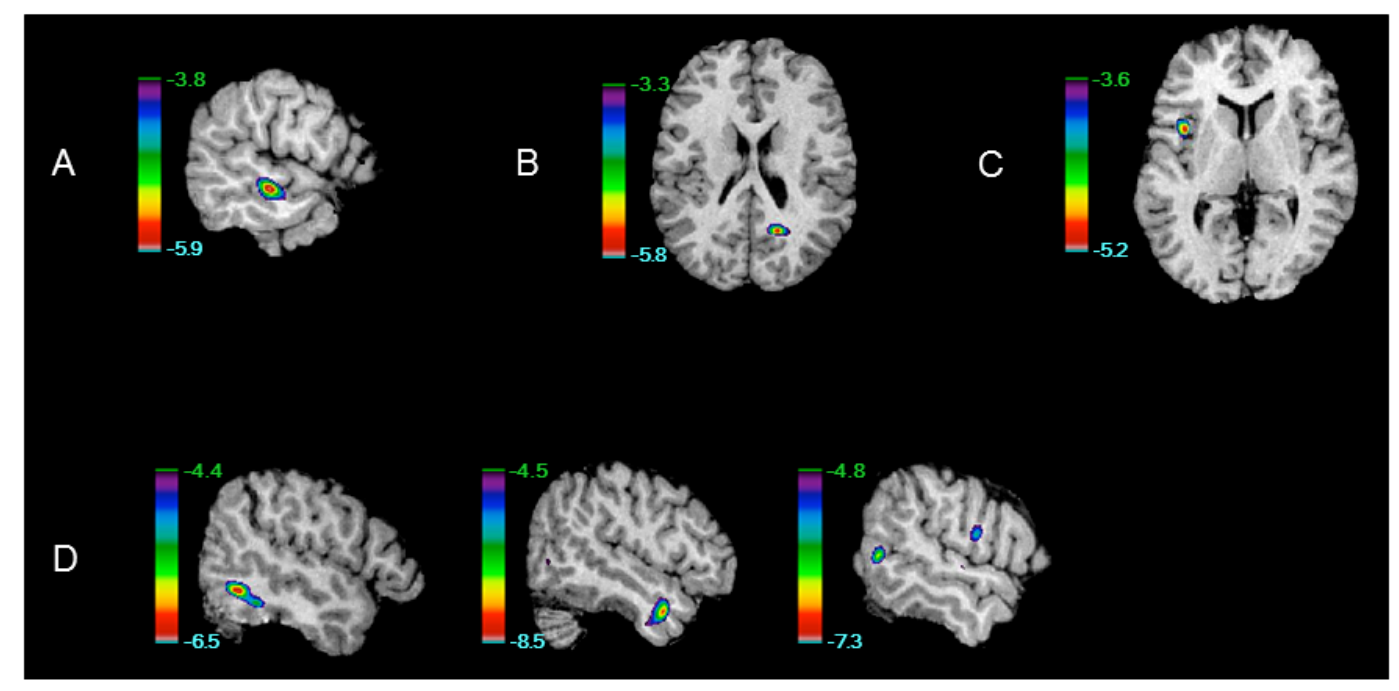

Figure 6. Extra-lesional GM decrease in four FCD patients (A-D). Areas of extralesional GM decrease are shown on the GM concentration z-score maps superimposed on the MRI. 


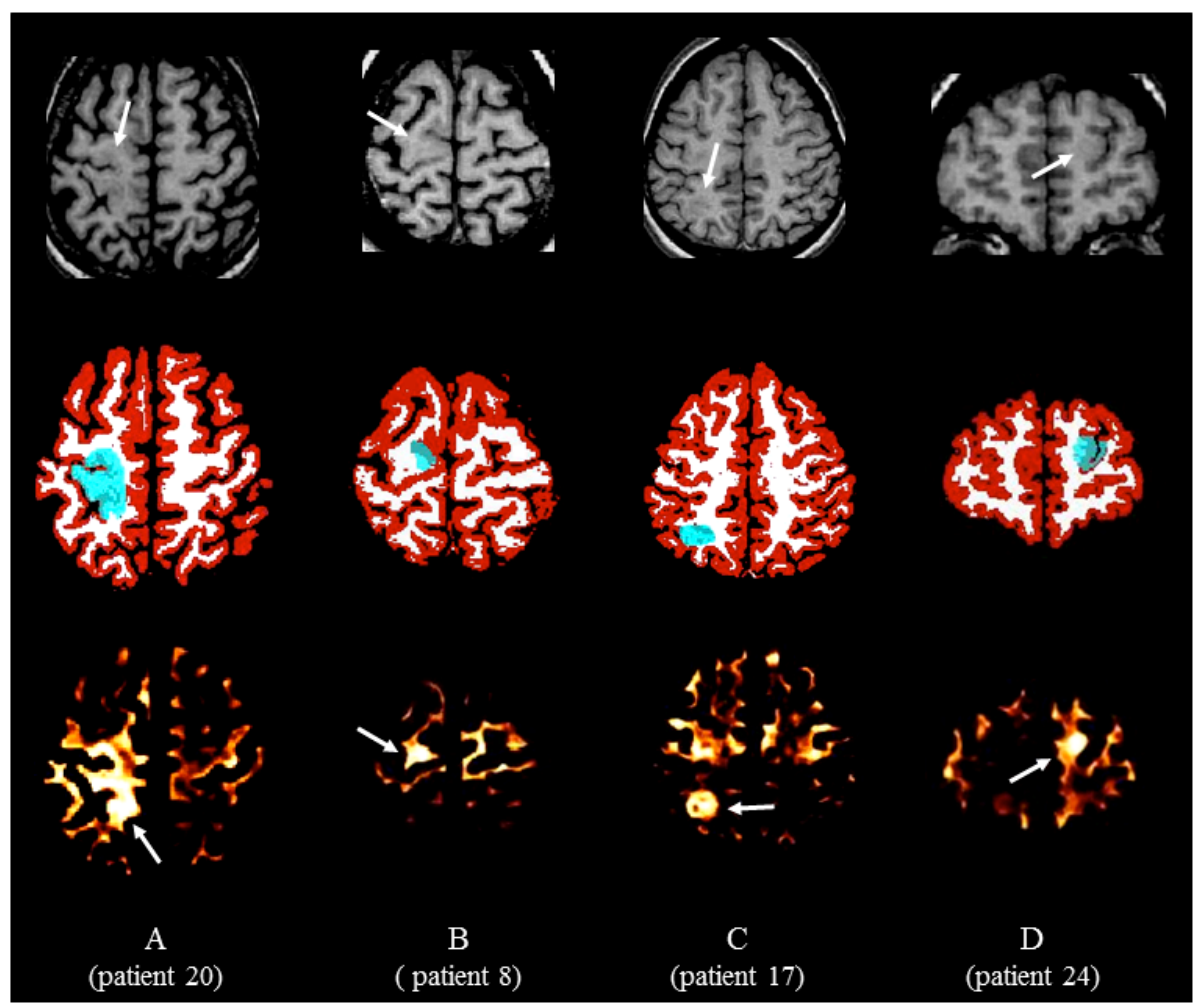

Figure 7. Four cases of undetected FCD due to lesion hyperintensity are presented (AD). For each patient, from top to bottom: T1-weighted MRI, the manual lesion label (blue) superimposed on the automatic MRI segmented map (GM in red, WM in white), and the relative intensity map (where brighter voxels correspond to higher relative intensity values). As shown on the segmented MRI map (middle panel), a large proportion of lesional voxels are misclassified as WM instead of GM. The mean relative intensity and the percentage of the lesion classified as GM in these patients are presented in Table 1. 


\section{References}

Antel S.B., Bernasconi A., Bernasconi N., Collins D.L., Kearney R.E., Shinghal R., Arnold D.L., 2002. Computational models of MRI characteristics of focal cortical dysplasia improve lesion detection. Neuroimage 17, 1755-1760.

Antel S.B., Collins D.L., Bernasconi N., Andermann F., Shinghal R., Kearney R.E., Arnold D.L., Bernasconi A., 2003. Automated detection of focal cortical dysplasia lesions using computational models of their MRI characteristics and texture analysis. Neuroimage 19, 1748-1759.

Ashburner J., Friston K.J., 2000. Voxel-based morphometry--the methods. Neuroimage $11,805-821$.

Barkovich A.J., Kuzniecky R.I., 1996. Neuroimaging of focal malformations of cortical development. J Clin Neurophysiol 13, 481-494.

Barkovich A.J., Kuzniecky R.I., Jackson G.D., Guerrini R., Dobyns W.B., 2001. Classification system for malformations of cortical development: update 2001. Neurology 57, 2168-2178.

Barth P.G., 1987. Disorders of neuronal migration. Can.J.Neurol.Sci. 14, 1-16.

Bastos A.C., Comeau R.M., Andermann F., Melanson D., Cendes F., Dubeau F., Fontaine S., Tampieri D., Olivier A., 1999. Diagnosis of subtle focal dysplastic lesions: curvilinear reformatting from three-dimensional magnetic resonance imaging. Ann Neurol 46, 88-94.

Bernasconi A., Antel S.B., Collins D.L., Bernasconi N., Olivier A., Dubeau F., Pike G.B., Andermann F., Arnold D.L., 2001. Texture analysis and morphological processing of magnetic resonance imaging assist detection of focal cortical dysplasia in extratemporal partial epilepsy. Ann Neurol 49, 770-775.

Bronen R.A., Spencer D.D., Fulbright R.K., 2000. Cerebrospinal fluid cleft with cortical dimple: MR imaging marker for focal cortical dysgenesis. Radiology 214, 657-663.

Cohen-Gadol A.A., Ozduman K., Bronen R.A., Kim J.H., Spencer D.D., 2004. Longterm outcome after epilepsy surgery for focal cortical dysplasia. J Neurosurg 101, 5565.

Collins D.L., Neelin P., Peters T.M., Evans A.C., 1994. Automatic 3D intersubject registration of MR volumetric data in standardized Talairach space. J Comput Assist Tomogr 18, 192-205. 
Colliot O., Antel S., Naessens V., Bernasconi N., Bernasconi A., 2004. Quantitative MRI profiling of cortical dysplasia. Epilepsia 45, 286.

Colombo N., Tassi L., Galli C., Citterio A., Lo Russo G., Scialfa G., Spreafico R., 2003. Focal cortical dysplasias: MR imaging, histopathologic, and clinical correlations in surgically treated patients with epilepsy. AJNR Am J Neuroradiol 24, 724-733.

Engel J., Jr., 1996. Surgery for seizures. N.Engl.J.Med. 334, 647-652.

Engel J., Jr., Van Ness P.C., Rasmussen T., Ojemann L.M., 1993. Outcome with respect to epileptic seizures. In: Engel J., Jr., editor. Surgical treatment of the epilepsies, Raven, New York, pp. 609-621.

Fauser S., Schulze-Bonhage A., Honegger J., Carmona H., Huppertz H.J., Pantazis G., Rona S., Bast T., Strobl K., Steinhoff B.J., Korinthenberg R., Rating D., Volk B., Zentner J., 2004. Focal cortical dysplasias: surgical outcome in 67 patients in relation to histological subtypes and dual pathology. Brain 127, 2406-2418.

Francione S., Vigliano P., Tassi L., Cardinale F., Mai R., Lo Russo G., Munari C., 2003. Surgery for drug resistant partial epilepsy in children with focal cortical dysplasia: anatomical-clinical correlations and neurophysiological data in 10 patients. J Neurol Neurosurg Psychiatry 74, 1493-1501.

Frater J.L., Prayson R.A., Morris I.H., Bingaman W.E. Surgical pathologic findings of extratemporal-based intractable epilepsy: a study of 133 consecutive resections. In: Arch Pathol Lab Med, 2000: 545-549.

Gambardella A., Palmini A., Andermann F., Dubeau F., Da Costa J.C., Quesney L.F., Andermann E., Olivier A., 1996. Usefulness of focal rhythmic discharges on scalp EEG of patients with focal cortical dysplasia and intractable epilepsy. Electroencephalogr Clin Neurophysiol 98, 243-249.

Gomez-Anson B., Thom M., Moran N., Stevens J., Scaravilli F., 2000. Imaging and radiological-pathological correlation in histologically proven cases of focal cortical dysplasia and other glial and neuronoglial malformative lesions in adults. Neuroradiology 42, 157-167.

Grant P.E., Vingneron D.B., Barkovich A.J., 1998. High-resolution imaging of the brain. Magn Reson Imaging Clin N Am 1, 139-154.

Jacobs K.M., Hwang B.J., Prince D.A., 1999. Focal epileptogenesis in a rat model of polymicrogyria. J.Neurophysiol. 81, 159-173. 
Kraemer M., Roth-Haerer A., Bruehl C., Luhmann H.J., Witte O.W., 2001. Metabolic and electrophysiological alterations in an animal model of neocortical neuronal migration disorder. Neuroreport 12, 2001-2006.

Kral T., Clusmann H., Blumcke I., Fimmers R., Ostertun B., Kurthen M., Schramm J., 2003. Outcome of epilepsy surgery in focal cortical dysplasia. J Neurol Neurosurg Psychiatry 74, 183-188.

Lee B.C., Schmidt R.E., Hatfield G.A., Bourgeois B., Park T.S., 1998. MRI of focal cortical dysplasia. Neuroradiology 40, 675-683.

Lerch J.P., Pruessner J.C., Zijdenbos A., Hampel H., Teipel S.J., Evans A.C., 2004. Focal Decline of Cortical Thickness in Alzheimer's Disease Identified by Computational Neuroanatomy. Cereb Cortex.

Mangin J.F., Frouin V., Bloch I., Regis J., Lopez-Krahe J., 1995. From 3D magnetic resonance images to structural representations of the cortex topography using topology preserving deformations. J Math Imag and Vision 5, 297-318.

Mehta S., Grabowski T.J., Trivedi Y., Damasio H., 2003. Evaluation of voxel-based morphometry for focal lesion detection in individuals. Neuroimage 20, 1438-1454.

Miller S.P., Li L.M., Cendes F., Tasch E., Andermann F., Dubeau F., Arnold D.L., 2000. Medial temporal lobe neuronal damage in temporal and extratemporal lesional epilepsy. Neurology 54, 1465-1470.

Mummery C.J., Patterson K., Price C.J., Ashburner J., Frackowiak R.S., Hodges J.R., 2000. A voxel-based morphometry study of semantic dementia: relationship between temporal lobe atrophy and semantic memory. Ann Neurol 47, 36-45.

Muntaner L., Perez-Ferron J.J., Herrera M., Rosell J., Taboada D., Climent S., 1997. MRI of a family with focal abnormalities of gyration. Neuroradiology 39, 605-608.

Palmini A., Gambardella A., Andermann F., Dubeau F., Da Costa J.C., Olivier A., Tampieri D., Gloor P., Quesney L.F., Andermann E., al. e., 1995. Intrinsic epileptogenicity of human dysplastic cortex as suggested by corticography and surgical results. Ann Neurol 37, 476-487.

Prayson R.A., Frater J.L. Cortical dysplasia in extratemporal lobe intractable epilepsy: a study of 52 cases. In: Ann Diagn Pathol, 2003: 139-146.

Prayson R.A., Spreafico R., Vinters H.V., 2002. Pathologic characteristics of the cortical dysplasias. Neurosurg.Clin.N.Am. 13, 17-25. 
Rakic P., 1988. Defects of neuronal migration and the pathogenesis of cortical malformations. Prog.Brain Res. 73, 15-37.

Raymond A.A., Fish D.R., 1996. EEG features of focal malformations of cortical development. J.Clin.Neurophysiol. 13, 495-506.

Raymond A.A., Fish D.R., Sisodiya S.M., Alsanjari N., Stevens J.M., Shorvon S.D., 1995. Abnormalities of gyration, heterotopias, tuberous sclerosis, focal cortical dysplasia, microdysgenesis, dysembryoplastic neuroepithelial tumor and dysgenesis of archicortex in epilepsy. Clinical, EEG and neuroimaging features in 100 adult patients. Brain 118, 629-660.

Redecker C., Hagemann G., Witte O.W., Marret S., Evrard P., Gressens P., 1998. Longterm evolution of excitotoxic cortical dysgenesis induced in the developing rat brain. Brain Res.Dev.Brain Res. 109, 109-113.

Riviere D., Mangin J.F., Papadopoulos-Orfanos D., Martinez J.M., Frouin V., Regis J., 2002. Automatic recognition of cortical sulci of the human brain using a congregation of neural networks. Med Image Anal 6, 77-92.

Russo G.L., Tassi L., Cossu M., Cardinale F., Mai R., Castana L., Colombo N., Bramerio M., 2003. Focal cortical resection in malformations of cortical development. Epileptic Disord 5 Suppl 2, S115-123.

Sailer M., Fischl B., Salat D., Tempelmann C., Schonfeld M.A., Busa E., Bodammer N., Heinze H.J., Dale A., 2003. Focal thinning of the cerebral cortex in multiple sclerosis. Brain 126, 1734-1744.

Salmond C.H., Ashburner J., Vargha-Khadem F., Connelly A., Gadian D.G., Friston K.J., 2002. Distributional assumptions in voxel-based morphometry. Neuroimage 17, 1027-1030.

Sisodiya S.M., 2000. Surgery for malformations of cortical development causing epilepsy. Brain 123, 1075-1091.

Sisodiya S.M., 2004. Malformations of cortical development: burdens and insights from important causes of human epilepsy. Lancet Neurol 3, 29-38.

Sisodiya S.M., Free S., Stevens J.M., Fish D.R., Shorvon S.D., 1995. Widespread cerebral structural changes in patients with cortical dysgenesis and epilepsy. Brain 118, 1039-1050. 
Sisodiya S.M., Free S.L., 1997. Disproportion of cerebral surface areas and volumes in cerebral dysgenesis. MRI-based evidence for connectional abnormalities. Brain 120 (Pt 2), 271-281.

Sled J.G., Zijdenbos A.P., Evans A.C., 1998. A nonparametric method for automatic correction of intensity nonuniformity in MRI data. IEEE Trans Med Imaging 17, 8797.

Tassi L., Colombo N., Garbelli R., Francione S., Lo R.G., Mai R., Cardinale F., Cossu M., Ferrario A., Galli C., Bramerio M., Citterio A., Spreafico R., 2002. Focal cortical dysplasia: neuropathological subtypes, EEG, neuroimaging and surgical outcome. Brain 125, 1719-1732.

Tassi L., Pasquier B., Minotti L., Garbelli R., Kahane P., Benabid A.L., Battaglia G., Munari C., Spreafico R., 2001. Cortical dysplasia: electroclinical, imaging, and neuropathologic study of 13 patients. Epilepsia 42, 1112-1123.

Taylor D.C., Falconer M.A., Bruton C.J., Corsellis J.A.N., 1971. Focal dysplasia of the cerebral cortex in epilepsy. J Neurol Neurosurg Psychiatry 34, 369-387.

Usui N., Matsuda K., Mihara T., Tottori T., Ohtsubo T., Baba K., Matsuyama N., Inoue Y., Yagi K., Kajita Y., Yoshida J., 2001a. MRI of cortical dysplasia--correlation with pathological findings. Neuroradiology 43, 830-837.

Usui N., Matsuda K., Mihara T., Tottori T., Ohtsubo T., Baba K., Matsuyama N., Inoue Y., Yagi K., Kajita Y., Yoshida J., 2001b. MRI of cortical dysplasia--correlation with pathological findings. Neuroradiology 43, 830-837.

Wilke M., Kassubek J., Ziyeh S., Schulze-Bonhage A., Huppertz H.J., 2003. Automated detection of gray matter malformations using optimized voxel-based morphometry: a systematic approach. Neuroimage 20, 330-343.

Woermann F.G., Free S.L., Koepp M.J., Ashburner J., Duncan J.S., 1999. Voxel-byvoxel comparison of automatically segmented cerebral gray matter--A raterindependent comparison of structural MRI in patients with epilepsy. Neuroimage. 10, 373-384.

Yagishita A., Arai N., Maehara T., Shimizu H., Tokumaru A.M., Oda M., 1997. Focal cortical dysplasia: appearance on MR images. Radiology 203, 553-559.

Zijdenbos A.P., Forghani R., Evans A.C. Automatic quantification of ms lesions in 3D MRI brain data sets: validation of INSECT. In: Wells W.M., Colchester A.C., Delp S., editors. Medical Image Computing and Computer-assisted Intervention 
(MICCAI); 1998 1998; Cambridge MA, USA, Proceedings (Lecture notes in Computer Science, 1496): Springer-Verlag Telos, 1998: 439-448. 